\title{
Comparison of Volatile Compounds in Two Brandies Using HS-SPME Coupled with GC-O, GC-MS and Sensory Evaluation
}

\author{
Y.P. Zhao ${ }^{1 *}$, L. Wang ${ }^{1}$, J.M. Li ${ }^{2}$, G.R. Pei ${ }^{1}$ and Q.S. Liu ${ }^{1}$ \\ (1) Institute of Food Science and Engineering, Yantai University, Yantai, Shandong, PR China, 264005 \\ (2) Technology Center of Changyu Pioneer Wine Co. Ltd., Yantai, Shandong, PR China, 264005
}

Submitted for publication: May 2010

Accepted for publication: July 2010

Key words: Brandy, gas chromatography-mass spectrometry, gas chromatography-olfactometry, sensory evaluation

\begin{abstract}
The aim of this study was to compare the volatile compounds between Changyu XO and Hennessy XO. Sensory evaluation was performed by a panel of tasters. Qualitative and semi-quantitative analysis was achieved by headspace solid phase micro-extraction (HS-SPME), coupled with gas chromatography-mass spectrometry (GCMS) and gas chromatography-olfactometry (GC-O). A total of 160 volatile compounds were identified in the two brands of brandy. Of these, 118 compounds were common to both Changyu XO and Hennessy XO; 18 compounds were specific to Changyu XO and 24 were specific to Hennessy XO. A total of 85 aroma compounds responsible for brandy flavour were identified by GC-O, of which 68 were common to both brands, while seven and ten were specific to Changyu XO and Hennessy XO, respectively. The study provided detailed information about the compounds responsible for the characteristic flavour of specific brandies. According to statistical analysis, significant differences were recorded between Changyu XO and Hennessy XO. Most volatile compounds in Changyu XO occurred at lower concentrations than those in Hennessy XO. Based on sensory evaluation analysis, the floral, alcohol and rancid aroma descriptors achieved higher scores in Changyu XO and Hennessy XO, while the lime aroma seemed specific to Hennessy XO. Herb and almond aromas were specific to Changyu XO.
\end{abstract}

\section{INTRODUCTION}

Hennessy XO, a typical French spirit liquor, is famous for its premium quality and Changyu $\mathrm{XO}$, a well-known Chinese brandy, is produced in Yantai (China). Yantai is located at the same latitude as Bordeaux (France) and is one of the largest grape growing regions in Asia. With brandy having become more popular in China over recent decades, its characteristic and distinct flavour began to receive closer scrutiny from the consumer.

In general, brandy is a distilled product of fermented grapes matured in oak barrels, with hundreds of volatile compounds developing over a period. Several authors (Onishi et al., 1978; Pérez-Coello et al., 1995; Caldeira et al., 2002; Ledauphin et al., 2004; Caldeira et al., 2006; Go'mez-M1'guez et al., 2007) have studied the chemical modifications in different brandies. The volatile compounds include various chemical classes, such as hydrocarbons, alcohols, esters, acids, ketones, aldehydes, and nitrogen- and sulphur-containing compounds. These are all volatile compounds derived from each successive stage of the production process and contribute to the aroma of brandies. Ledauphin et al. (2004) identified more than 300 volatile compounds in freshly distilled Cognac and Calvados by preparative separations coupled with GC-MS. Ferrari et al. (2004) identified 150 volatile compounds in freshly distilled Cognac by GC-MS. Of these, 34 are responsible for the odours. Zhao et al. (2009b) identified 144 volatile compounds in
Changyu and Hennessy brandies by HS-SPME coupled with GC-MS

Volatile compounds are produced by grapes and in wines during fermentation, distillation and ageing in oak barrels (Milicevic et al., 2002; Ferrari et al., 2004; Go'mez-M1'guez et al., 2007; Van Jaarsveld et al., 2009a,b,c). Hydrocarbons are formed by the raw materials and the original process (Ferrari et al., 2004; Fan \& Qian, 2006). Fusel alcohols, the most abundant alcohols, are formed during fermentation from amino acids through decarboxylation and deamination (Ferrari et al., 2004; Fan \& Qian, 2006). Esters, mainly formed during fermentation, constitute the most abundant chemical class of aroma compounds in brandies (Ferrari et al., 2004; Fan \& Qian, 2006). Ketones and aldehydes are derived from the fermentation and distillation processes (Fan \& Qian, 2006). Some aroma compounds may form by direct extraction of molecules from the oak and by degradation of oak macromolecules into aroma compounds (Ferrari et al., 2004; Fan \& Qian, 2006).

The aim of this study was to compare the volatile compounds of Changyu XO and Hennessy XO by GC-MS coupled with GC-O and sensorial analysis to explore the key components resulting in the volatile difference between the two brands.

\section{MATERIALS AND METHODS \\ Brandy samples}

Three Changyu XO samples were provided by the Changyu

*Corresponding author: water15689@163.com [Tel.: +86-535-6902501, Fax: +86-535-6902063]

Acknowledgements: This work was financially supported by The Key Technologies R \& D Program of Shandong Province (200910506004) 
Pioneer Wine Co. Ltd. (Yantai, China), which included CXO1 (bottledin2000,730mL,40\%v/vethanol), CXO2(bottledin 2008 , $730 \mathrm{~mL}, 40 \% \mathrm{v} / \mathrm{v}$ ethanol) and CXO3 (bottled in 2004,730 mL, $40 \% \mathrm{v} / \mathrm{v}$ ethanol). Three Hennessy XO samples were purchased from a local store; these included HXO1 (bottled in 1998, 700 $\mathrm{mL}, 40 \% \mathrm{v} / \mathrm{v}$ ethanol), HXO2 (bottled in 2005, 700mL, 40\% v/v ethanol) and HXO3 (bottled in 2006, $700 \mathrm{~mL}, 40 \% \mathrm{v} / \mathrm{v}$ ethanol).

\section{Reagents}

Sodium chloride was purchased from China National Pharmaceutical Ground Corporation (Shanghai, China). Methanol was purchased from Merck Chemical Co. Inc. (Shanghai, China). All standards, including 3-octanol (inner standard) and the $\mathrm{C} 7$ to $\mathrm{C} 30$ alkanes were obtained from Aldrich-Sigma Chemical Co. (Shanghai, China).

\section{Sensory analysis}

Sensory evaluation was performed by a panel of 12 members (six males and six females), trained for primary sensory analyses. The aroma descriptors previously selected by the panel were e.g. floral, woody, rancid, caramel, burned/toasted, rose, butter, fruity, green, tails and glue/varnish. Brandy quality was assessed according to odour and aroma balance. The panel scored the samples according to a structured scale $(0$, no perception, 5 to 10,10 to 15,15 to 20 , and above 20 ).

\section{HS-SPME parameters}

The operating factors for GC-MS analysis (Howard et al., 2005), including extraction time (10 $\mathrm{min}, 20 \mathrm{~min}, 30 \mathrm{~min}$ and $40 \mathrm{~min})$, extraction temperature $\left(30^{\circ} \mathrm{C}, 40^{\circ} \mathrm{C}, 50^{\circ} \mathrm{C}\right.$ and $\left.60^{\circ} \mathrm{C}\right)$, ethanol concentration $(5 \%, 10 \%, 15 \%$ and $35 \%, \mathrm{v} / \mathrm{v})$, and salt added $(0.5 \mathrm{~g}, 1.0 \mathrm{~g}, 1.5 \mathrm{~g}$ and $2.0 \mathrm{~g})$, were optimised by the $4^{5}$ four-level full-factorial design (FFD) . The best condition was at $50^{\circ} \mathrm{C}$ for $30 \mathrm{~min}$, with $2.0 \mathrm{~g}$ salt added and the alcohol content adjusted to $10 \%(\mathrm{v} / \mathrm{v})$.

\section{HS-SPME analysis}

A 50/30 $\mu \mathrm{m}$ DVB/CAR/PDMS fibre (Supelco, Inc., Bellefonte, PA) was used for aroma extraction. Each liquor sample was diluted with deionised water to a final concentration of $10 \%$ $(\mathrm{v} / \mathrm{v})$ ethanol. The total volume $[5 \mathrm{~mL}$ solution and $5 \mu \mathrm{L}$ inner standard (3-octanol, $640.56 \mathrm{mg} / \mathrm{L}$ )], was transferred into a 20 $\mathrm{mL}$ vial. The diluted sample was saturated with sodium chloride and the vial tightly capped with a silicon septum. The sample was equilibrated at $50^{\circ} \mathrm{C}$ in a thermostatic bath for $10 \mathrm{~min}$ and extracted at the same temperature for $30 \mathrm{~min}$, under stirring. After extraction, the fibre was inserted into the injection port of the $\mathrm{GC}\left(250^{\circ} \mathrm{C}\right)$.

\section{GC-MS analysis}

GC-MS analysis was performed using a Shimadzu GC 2010 mass selective detector. Samples were analysed on a DB-Wax column. The carrier gas was helium at a constant flow rate of $1.2 \mathrm{~mL} / \mathrm{min}(39 \mathrm{~cm} / \mathrm{s})$. The oven temperature was kept at $50^{\circ} \mathrm{C}$ for two $\mathrm{min}$, followed by an increase of $4^{\circ} \mathrm{C} / \mathrm{min}$ to a final temperature of $250^{\circ} \mathrm{C}$ and kept at the final temperature for three min. The splitless injector port was set to $250^{\circ} \mathrm{C}$. The mass spectrometer was operated with the electron impact (EI) at $70 \mathrm{eV}$ as ionisation potential. The injector temperature was kept constant at $250^{\circ} \mathrm{C}$. The transfer line was kept at $250^{\circ} \mathrm{C}$. A mass range from m/z 32-500 (2 scan/s) was recorded in full scan mode, without solvent delay.

\section{Qualitative and quantitative analysis Qualitative analysis}

Mass spectra of unknown compounds were compared with those in the National Institute of Standards and Technology (NIST) 98 MS database or a "private" database. Retention indices (RI) were calculated in accordance with a modified Kovats method (Ledauphin et al., 2004). A standard mixture of paraffin homologues $\mathrm{C}_{7}$ to $\mathrm{C}_{30}$ was prepared. The sample and the hydrocarbon standard mixture were co-injected into the GC, and the retention times were used to calculate the RI. Identification of unknown compounds was achieved by comparing the mass spectra and RI of the standards or retention indices from literature (RIL) (Fan and Qian, 2006).

\section{Semi-quantitative analysis}

Semi-quantitative analysis was used to analyse the volatiles in brandy. An internal standard solution (3-octanol, $640.56 \mathrm{mg} / \mathrm{L}$ ) was individually prepared in ethanol prior to dilution. Selective ion monitoring (SIM) was used for the integrations of all chromatogram peaks. And the semi-quantitative concentrations of volatiles in brandies were calculated according to the method proposed by Zhao et al. (2009b), as follows:

$\begin{aligned} & \text { Semiquantitative concentrations } \\ & \text { concentration }\end{aligned} \quad \frac{\text { peak area }}{\text { IS peak area }} \times$ IS

\section{Statistics analysis}

Mean peak areas and standard deviations from replicate analyses were calculated and treatment variables were compared using the Student T test (Steel \& Torrie, 1980).

\section{RESULTS AND DISCUSSION}

\section{Sensory analysis}

The tasting panel provided an assessment of brandy samples. The observed relative standard deviations (RSD) from the mean aroma descriptor intensities varied within the range of 2.0 to $4.0 \%$. The aroma profiles (Fig. 1) are characteristic for each brandy sample. The three Hennessy XO samples showed similar profiles in which the floral, alcohol and rancid aroma descriptors had higher scores, followed by fruity, grass, hay, lime, tails, and roast aromas. As for three Changyu samples, their higher scores were the floral, alcohol and rancid aromas, followed by fruity, grass, hay, tails, herb, almond, and roast aromas. The lime aroma seemed specific to Hennessy XO samples, whereas the herb and almond aromas were specific to Changyu XO samples. In addition, the greater differences were found in the aroma profiles of the three Changyu samples.

\section{Identification of aroma volatile compounds}

The GC-MS analysis was performed to identify the volatile compounds in the six brandies. The total chromatograms of volatiles in Changyu XO and Hennessy XO are shown in Fig. 2. The common volatiles in Changyu XO and Hennessy $\mathrm{XO}$ are listed in Table 1, whereas, the specific compounds in these samples are presented in Tables 2 and 3, respectively. A total of 184 compounds were identified in the six brandies, 
TABLE 1

Common volatile compounds in three Changyu samples and three Hennessy samples by GC-MS on a DB-Wax column.

\begin{tabular}{|c|c|c|c|c|c|c|c|c|c|c|c|c|}
\hline \multirow[t]{2}{*}{ RI } & \multirow[t]{2}{*}{ Compound $^{1}$} & \multirow[t]{2}{*}{ Descriptor } & \multirow[t]{2}{*}{ Identification ${ }^{2}$} & \multicolumn{3}{|c|}{$\begin{array}{c}\text { Mean concentration } \\
(\mathrm{mg} / \mathrm{L})^{3}\end{array}$} & \multirow[t]{2}{*}{ CV } & \multicolumn{3}{|c|}{$\begin{array}{c}\begin{array}{c}\text { Mean concentration } \\
(\mathrm{mg} / \mathrm{L})\end{array} \\
\end{array}$} & \multirow[t]{2}{*}{ CV } & \multirow[t]{2}{*}{ Judge $^{4}$} \\
\hline & & & & CXO1 & $\mathrm{CXO3}$ & CXO2 & & HXO1 & HXO2 & HXO3 & & \\
\hline & Esters & & & & & & & & & & & \\
\hline$>900$ & ethyl acetate & pineapple & MS,A,RI & 2.346 & 2.889 & 2.880 & 0.11 & 1.481 & 1.640 & 1.246 & 0.14 & $* *$ \\
\hline 1022 & ethyl butanoate & fruity & MS,A,RI & 0.026 & 0.028 & 0.021 & 0.14 & 0.060 & n.i. & 0.076 & 0.17 & $* * *$ \\
\hline 1040 & ethyl 2-methylbutanoate & & $\mathrm{MS}, \mathrm{RIL}^{\mathrm{a}}$ & n.i. & 0.060 & 0.059 & 0.02 & 0.122 & 0.148 & 0.190 & 0.22 & $* * *$ \\
\hline 1051 & ethyl 3-methylbutanoate & apple & MS,A,RI & 0.010 & 0.020 & 0.014 & 0.31 & 0.059 & 0.045 & 0.050 & 0.14 & $* * *$ \\
\hline 1127 & 3-methylbutyl acetate & banana & MS,A,RI & 0.286 & n.i. & 0.446 & 0.31 & 0.139 & 0.701 & 0.820 & 0.66 & n.s. \\
\hline 1230 & ethyl hexanoate & fruity, wine & MS,A,RI & 0.340 & 0.262 & 0.331 & 0.14 & 1.569 & 1.181 & 1.232 & 0.16 & $* * *$ \\
\hline 1240 & ethyl orthoformate $\mathrm{e}^{\mathrm{T}}$ & & MS & 0.063 & 0.084 & 0.076 & 0.14 & 0.180 & 0.159 & 0.210 & 0.14 & $* * *$ \\
\hline 1261 & hexyl acetate & fruity, sweet & MS,A,RI & 0.038 & 0.035 & 0.013 & 0.47 & 0.007 & 0.005 & n.i. & 0.16 & $* * *$ \\
\hline 1301 & methyl 2-hydroxypropanoate ${ }^{\mathrm{T}}$ & & MS & 0.003 & 0.007 & 0.004 & 0.46 & 0.007 & 0.010 & 0.016 & 0.42 & $* * *$ \\
\hline 1321 & ethyl heptanoate & fruity & MS,A,RI & 0.154 & 0.025 & 0.039 & 0.97 & 0.018 & 0.018 & 0.015 & 0.11 & $* * *$ \\
\hline 1329 & ethyl 3-ethoxypropanoate ${ }^{\mathrm{T}}$ & & MS & n.i. & 0.025 & 0.018 & 0.21 & 0.026 & 0.063 & 0.045 & 0.41 & $* * *$ \\
\hline 1341 & ethyl 2-hydroxypropanoate & fruity & MS,A,RI & 0.126 & 0.109 & 0.129 & 0.09 & 0.123 & 0.126 & 0.115 & 0.04 & $* * *$ \\
\hline 1411 & ethyl 2-hydroxy-3-methylbutanoate & floral & MS,A,RI & 0.005 & 0.009 & 0.010 & 0.33 & 0.009 & 0.022 & 0.012 & 0.48 & $* * *$ \\
\hline 1414 & ethyl octanoate & cooked fruity & MS,A,RI & 26.725 & 28.339 & 27.985 & 0.03 & 29.336 & 27.237 & 28.313 & 0.04 & n.s. \\
\hline 1442 & 3-methylbutyl hexanoate ${ }^{\mathrm{T}}$ & & MS & 0.003 & 0.003 & 0.003 & 0.00 & 0.052 & 0.041 & 0.050 & 0.13 & $* * *$ \\
\hline 1470 & ethyl diethoxyacetate & floral & MS,A,RI & 0.004 & 0.007 & 0.007 & 0.27 & 0.010 & 0.010 & 0.009 & 0.08 & $* * *$ \\
\hline 1533 & ethyl nonanoate & fruity & MS,A,RI & 0.028 & 0.031 & 0.031 & 0.08 & 0.052 & 0.062 & 0.058 & 0.08 & $* * *$ \\
\hline 1550 & ethyl 2-hydroxyhexanoate & & MS, RIL ${ }^{b}$ & 0.041 & 0.025 & 0.028 & 0.27 & 0.014 & 0.030 & 0.018 & 0.39 & $* * *$ \\
\hline 1567 & 3-methylbutyl 2-hydroxypropanoate & & MS, RIL ${ }^{b}$ & 0.058 & 0.033 & 0.037 & 0.32 & 0.021 & n.i. & n.i. & --- & n.s. \\
\hline 1577 & diethyl propanedioate & & MS, RIL ${ }^{\mathrm{a}}$ & 0.008 & 0.016 & 0.014 & 0.33 & 0.008 & 0.026 & 0.016 & 0.55 & $* * *$ \\
\hline 1584 & methyl decanoate & & MS, RIL ${ }^{a}$ & 0.013 & 0.010 & 0.010 & 0.13 & 0.037 & 0.030 & 0.029 & 0.13 & $* * *$ \\
\hline 1610 & ethyl 4-oxopentanoate & grape & $\mathrm{MS}, \mathrm{A}, \mathrm{RIL}^{\mathrm{b}}$ & 0.009 & 0.007 & n.i. & 0.24 & 0.013 & 0.033 & 0.020 & 0.46 & $* * *$ \\
\hline 1648 & ethyl decanoate & fruity & MS,A,RI & 10.247 & 10.155 & 9.892 & 0.02 & 11.506 & 12.005 & 11.821 & 0.02 & $* * *$ \\
\hline 1649 & 2-methylbutyl octanoate & fruity & $\mathrm{MS}, \mathrm{A}, \mathrm{RIL}^{\mathrm{a}}$ & 0.136 & 0.110 & 0.134 & 0.11 & 0.355 & 0.338 & 0.361 & 0.03 & $* * *$ \\
\hline 1680 & diethyl succinate & fruity & MS,A,RI & 0.289 & 0.282 & 0.327 & 0.08 & 0.472 & 0.345 & 0.475 & 0.17 & $* * *$ \\
\hline 1685 & ethyl dec-9-enoate & fruity & MS,A,RI & 0.062 & 0.118 & 0.112 & 0.32 & 0.255 & 0.206 & 0.201 & 0.13 & $* * *$ \\
\hline 1705 & methyl undecanoate ${ }^{\mathrm{T}}$ & & MS & 0.005 & n.i. & 0.003 & 0.47 & 0.016 & 0.025 & 0.059 & 0.69 & $* * *$ \\
\hline 1747 & propyl decanoate & fruity & $\mathrm{MS}, \mathrm{A}, \mathrm{RIL}^{\mathrm{a}}$ & 0.020 & 0.018 & 0.026 & 0.20 & 0.142 & 0.138 & 0.131 & 0.04 & $* * *$ \\
\hline 1771 & methyl salicylate & pine & MS,A,RI & 0.024 & 0.017 & 0.017 & 0.20 & 0.062 & 0.063 & 0.081 & 0.16 & $* * *$ \\
\hline 1775 & diethyl pentanedioate & & MS, RIL ${ }^{a}$ & n.i. & 0.004 & n.i. & --- & 0.030 & 0.029 & 0.032 & 0.05 & n.s. \\
\hline 1800 & ethyl 2-methylpropyl succinate & fruity & MS,A,RIL ${ }^{a}$ & 0.009 & 0.013 & 0.010 & 0.18 & n.i. & 0.091 & 0.080 & 0.09 & $* * *$ \\
\hline 1831 & ethyl 2,3-diethoxypropanoate & & MS, RIL ${ }^{\mathrm{c}}$ & n.i. & n.i. & 0.005 & --- & 0.025 & 0.032 & 0.039 & 0.22 & n.s. \\
\hline 1843 & ethyl dodecanoate & sweet, fruity & $\mathrm{MS}, \mathrm{A}, \mathrm{RIL}^{\mathrm{a}}$ & 14.235 & 15.849 & 15.167 & 0.05 & 17.332 & 15.731 & 15.967 & 0.05 & n.s. \\
\hline 1862 & isopentyl decanoate & & MS,RIL ${ }^{a}$ & 0.089 & 0.085 & 0.094 & 0.05 & 0.135 & 0.445 & 0.231 & 0.59 & $* *$ \\
\hline 1890 & diethyl hexanedioate ${ }^{\mathrm{T}}$ & & MS & n.i. & 0.012 & 0.013 & 0.07 & 0.020 & 0.025 & 0.023 & 0.12 & $* * *$ \\
\hline 2005 & diethyl pentanedioate & & $\mathrm{MS}, \mathrm{RIL}^{\mathrm{c}}$ & 0.024 & 0.014 & 0.020 & 0.24 & 0.022 & 0.076 & 0.075 & 0.53 & $* * *$ \\
\hline 2060 & ethyl tetradecanoate & & MS, RIL ${ }^{a}$ & 0.167 & 0.181 & 0.127 & 0.18 & 0.272 & 0.702 & 0.496 & 0.44 & $* *$ \\
\hline 2078 & isopentyl dadecanoate & & MS,RIL ${ }^{\mathrm{a}}$ & 0.018 & 0.013 & n.i. & 0.24 & 0.018 & 0.092 & n.i. & 0.94 & $* * *$ \\
\hline 2107 & ethyl 3-hydroxydecanoate ${ }^{\mathrm{T}}$ & & MS & 0.013 & 0.020 & n.i. & 0.28 & 0.045 & 0.048 & 0.052 & 0.08 & $* * *$ \\
\hline 2112 & diethyl octanedioate & fruity & $\mathrm{MS}, \mathrm{A}, \mathrm{RIL}^{\mathrm{c}}$ & 0.009 & 0.014 & 0.007 & 0.40 & 0.026 & 0.031 & 0.022 & 0.18 & $* * *$ \\
\hline 2139 & ethyl pentadecanoate & & MS, RIL ${ }^{\mathrm{b}}$ & 0.028 & 0.020 & 0.017 & 0.25 & 0.014 & 0.025 & n.i. & 0.38 & $* * *$ \\
\hline 2224 & isopropyl palmitate ${ }^{\mathrm{T}}$ & & MS & 0.105 & 0.146 & 0.083 & 0.29 & 0.135 & 0.154 & 0.109 & 0.17 & $* * *$ \\
\hline 2241 & ethyl hexadecanoate & fatty & $\mathrm{MS}, \mathrm{A}, \mathrm{RIL}^{\mathrm{a}}$ & 0.119 & 0.136 & 0.093 & 0.19 & 0.105 & 0.417 & 0.346 & 0.57 & $*$ \\
\hline 2251 & ethyl hexadec-9-enoate & fatty & $\mathrm{MS}, \mathrm{A}, \mathrm{RIL}^{\mathrm{a}}$ & 0.033 & 0.009 & n.i. & 0.80 & 0.041 & 0.037 & 0.034 & 0.09 & $* * *$ \\
\hline 2477 & ethyl octadecanoate & & MS,RIL ${ }^{a}$ & 0.004 & 0.003 & n.i. & 0.28 & 0.007 & 0.022 & 0.004 & 0.91 & $* * *$ \\
\hline 2479 & ethyl oleate & & $\mathrm{MS}, \mathrm{RIL}^{\mathrm{a}}$ & 0.009 & 0.009 & n.i. & 0.00 & 0.013 & 0.013 & 0.015 & 0.09 & $* * *$ \\
\hline 2531 & ethyl linoleate & & $\mathrm{MS}, \mathrm{RIL}^{\mathrm{a}}$ & 0.013 & 0.010 & n.i. & 0.16 & 0.022 & 0.029 & n.i. & 0.18 & $* * *$ \\
\hline & Total & & & 55.944 & 59.262 & 58.302 & 0.03 & 64.413 & 62.706 & 63.194 & 0.01 & n.s. \\
\hline
\end{tabular}




\begin{tabular}{|c|c|c|c|c|c|c|c|c|c|c|c|c|}
\hline \multirow[t]{2}{*}{ RI } & \multirow[t]{2}{*}{ Compound $^{1}$} & \multirow[t]{2}{*}{ Descriptor } & \multirow[t]{2}{*}{ Identification $^{2}$} & \multicolumn{3}{|c|}{$\begin{array}{c}\text { Mean concentration } \\
(\mathrm{mg} / \mathrm{L})^{3} \\
\end{array}$} & \multirow[t]{2}{*}{$\mathrm{CV}$} & \multicolumn{3}{|c|}{$\begin{array}{c}\text { Mean concentration } \\
(\mathrm{mg} / \mathrm{L})\end{array}$} & \multirow[t]{2}{*}{ CV } & \multirow[t]{2}{*}{ Judge } \\
\hline & & & & CXO1 & CXO3 & CXO2 & & HXO1 & HXO2 & HXO3 & & \\
\hline & Alcohols & & & & & & & & & & & \\
\hline 1029 & propan-1-ol & alcohol, fruity & MS,A,RI & 0.157 & 0.134 & 0.081 & 0.31 & 0.125 & 0.173 & 0.169 & 0.17 & $* * *$ \\
\hline 1094 & 2-methylpropanol & fusel & $\mathrm{MS}, \mathrm{A}, \mathrm{RI}$ & 1.729 & 2.822 & 2.879 & 0.26 & 2.569 & 2.682 & 2.971 & 0.08 & n.s. \\
\hline 1162 & butan-1-ol & alcohol, fruity & MS,A,RI & 0.018 & 0.018 & 0.016 & 0.09 & 0.023 & 0.020 & 0.021 & 0.08 & $* * *$ \\
\hline 1214 & 3-methylbutanol & fusel & MS,A,RI & 15.796 & 15.390 & 15.692 & 0.01 & 24.338 & 23.524 & 23.367 & 0.02 & $* * *$ \\
\hline 1254 & 1-pentanol & fruity & MS,A,RI & n.i. & 0.005 & 0.005 & 0.00 & 0.009 & 0.008 & 0.009 & 0.09 & $* * *$ \\
\hline 1296 & 4-methylpentanol & & $\mathrm{MS}, \mathrm{RIL}^{\mathrm{b}}$ & 0.004 & 0.005 & 0.005 & 0.16 & 0.008 & 0.011 & 0.009 & 0.16 & $* * *$ \\
\hline 1319 & heptan-2-ol & & MS, RIL ${ }^{b}$ & 0.018 & 0.030 & 0.025 & 0.24 & 0.035 & 0.046 & 0.050 & 0.17 & $* * *$ \\
\hline 1350 & hexan-1-ol & floral, green & $\mathrm{MS}, \mathrm{A}, \mathrm{RI}$ & 0.783 & 0.761 & 0.727 & 0.04 & 0.908 & 1.140 & 1.025 & 0.11 & $* * *$ \\
\hline 1360 & (E)-hex-3-en-1-ol & grass, leaf & MS,A,RI & 0.004 & 0.003 & 0.004 & 0.22 & 0.003 & 0.004 & 0.076 & 1.53 & $* * *$ \\
\hline 1371 & 3-ethoxypropanol & & $\mathrm{MS}, \mathrm{RIL}^{\mathrm{b}}$ & n.i. & 0.001 & n.i. & --- & n.i. & 0.003 & 0.005 & 0.47 & n.s. \\
\hline 1381 & (Z)-hex-3-en-1-ol & green & $\mathrm{MS}, \mathrm{A}, \mathrm{RI}^{\mathrm{b}}$ & 0.080 & 0.100 & 0.110 & 0.16 & 0.138 & 0.146 & 0.168 & 0.10 & $* * *$ \\
\hline 1409 & hex-2-en-1-ol & & MS, RIL ${ }^{\mathrm{a}}$ & 0.003 & 0.004 & 0.004 & 0.22 & 0.003 & 0.008 & 0.012 & 0.62 & $* * *$ \\
\hline 1451 & heptan-1-ol & & MS,RI & 0.007 & n.i. & n.i. & --- & 0.013 & 0.018 & n.i. & 0.22 & n.s. \\
\hline 1478 & 2-ethylhexanol & floral & MS,A,RI & 0.009 & 0.055 & 0.029 & 0.74 & 0.059 & 0.077 & 0.077 & 0.15 & $* * *$ \\
\hline 1561 & octan-1-ol & floral, green & MS,A,RI & 0.091 & 0.174 & 0.140 & 0.31 & 0.234 & 0.289 & 0.239 & 0.12 & $* * *$ \\
\hline 1649 & nonan-1-ol & floral & MS,A,RI & 0.025 & 0.034 & 0.034 & 0.17 & 0.050 & 0.067 & 0.077 & 0.21 & $* * *$ \\
\hline 1759 & decan-1-ol & fatty & MS,A,RI & 0.140 & 0.236 & 0.228 & 0.26 & 0.324 & 0.416 & 0.294 & 0.18 & $* * *$ \\
\hline 1869 & undecan-1-ol & & $\mathrm{MS}, \mathrm{RIL}^{\mathrm{a}}$ & 0.001 & 0.001 & 0.005 & 0.87 & n.i. & 0.005 & n.i. & --- & $* * *$ \\
\hline 1973 & dodecan-1-ol & rancid & MS,A,RIL ${ }^{\mathrm{a}}$ & 0.052 & 0.091 & 0.059 & 0.30 & 0.035 & 0.101 & 0.114 & 0.50 & $* *$ \\
\hline 2171 & tetradecan-1-ol & & MS,RIL ${ }^{a}$ & 0.087 & 0.096 & 0.071 & 0.15 & 0.094 & 0.144 & 0.810 & 1.14 & n.s. \\
\hline \multirow[t]{3}{*}{2369} & hexadecan-1-ol & & MS, RIL ${ }^{\mathrm{a}}$ & 0.029 & 0.047 & 0.030 & 0.29 & 0.055 & 0.033 & 0.073 & 0.38 & $* * *$ \\
\hline & Total & & & 19.033 & 20.007 & 20.144 & 0.03 & 29.023 & 28.915 & 29.566 & 0.01 & $* * *$ \\
\hline & Acids & & & & & & & & & & & \\
\hline 1441 & acetic acid & vinegar & MS,A,RI & 0.178 & 0.257 & 0.287 & 0.23 & 0.332 & 0.384 & 0.300 & 0.13 & $* * *$ \\
\hline 1627 & butanoic acid & rancid & MS,A,RI & n.i. & n.i. & 0.017 & --- & 0.024 & 0.025 & 0.031 & 0.14 & n.s. \\
\hline 1668 & 2/3-methylbutanoic acid & rancid & MS,A,RI & 0.009 & 0.016 & n.i. & 0.37 & 0.046 & 0.056 & 0.067 & 0.19 & $* * *$ \\
\hline 2066 & octanoic acid & fatty & MS,A,RI & 7.570 & 9.250 & 7.675 & 0.12 & 10.706 & 11.401 & 10.312 & 0.05 & n.s. \\
\hline 2154 & nonanoic acid & rancid & MS,A,RIL & 0.024 & 0.025 & 0.029 & 0.11 & 0.063 & 0.070 & 0.073 & 0.07 & $* * *$ \\
\hline 2248 & decanoic acid & rancid & MS,A,RIL ${ }^{\mathrm{a}}$ & 13.015 & 12.700 & 12.530 & 0.02 & 13.658 & 13.828 & 13.369 & 0.02 & $* *$ \\
\hline 2358 & 9-decenoic acid ${ }^{\mathrm{T}}$ & & MS & 0.003 & 0.010 & 0.008 & 0.57 & 0.029 & 0.014 & 0.022 & 0.33 & $* * *$ \\
\hline 2493 & dodecanoic acid & & MS, RIL ${ }^{\mathrm{a}}$ & 0.649 & 0.907 & 0.783 & 0.16 & 1.331 & 1.628 & 1.758 & 0.14 & $* * *$ \\
\hline 2680 & tetradecanoic acid & & MS,RIL ${ }^{\mathrm{a}}$ & 0.129 & 0.104 & 0.109 & 0.12 & 0.252 & 0.117 & 0.310 & 0.44 & $* * *$ \\
\hline 2931 & pentadecanoic acid & & MS,RI & 0.024 & 0.007 & 0.016 & 0.56 & 0.043 & n.i. & n.i. & --- & n.s. \\
\hline \multirow[t]{3}{*}{2975} & hexadecanoic acid ${ }^{\mathrm{T}}$ & & MS & 0.130 & 0.101 & 0.105 & 0.14 & 0.210 & 0.224 & 0.250 & 0.09 & $* * *$ \\
\hline & Total & & & 21.731 & 23.376 & 21.559 & 0.05 & 26.693 & 27.749 & 26.493 & 0.03 & * \\
\hline & Benzene derivatives & & & & & & & & & & & \\
\hline 1521 & $\begin{array}{l}\text { 3,4,4a,5,6,7-hexahydro-1,1,4a- } \\
\text { trimethyl-2(1H)-naphthalenone }\end{array}$ & & MS & 0.047 & 0.063 & 0.058 & 0.14 & 0.101 & 0.206 & 0.091 & 0.48 & $* * *$ \\
\hline 1664 & ethyl benzoate & floral & $\mathrm{MS}, \mathrm{A}, \mathrm{RI}$ & 0.038 & 0.018 & 0.042 & 0.39 & 0.048 & 0.054 & 0.060 & 0.11 & $* * *$ \\
\hline 1784 & ethyl 2-phenylacetate & honey & MS,A,RI & 0.014 & 0.020 & 0.016 & 0.16 & 0.094 & 0.072 & 0.105 & 0.18 & $* * *$ \\
\hline 1805 & 2-phenylethyl acetate & floral & MS,A,RI & 0.093 & 0.050 & 0.075 & 0.30 & 0.092 & 0.091 & 0.101 & 0.06 & $* * *$ \\
\hline 1879 & benzyl alcohol & floral & MS,A,RI & n.i. & 0.026 & 0.026 & 0.00 & n.i. & 0.022 & 0.028 & 0.15 & $* * *$ \\
\hline 1883 & ethyl benzenepropanoate & floral, fruity & $\mathrm{MS}, \mathrm{A}, \mathrm{RIL}^{\mathrm{d}}$ & 0.018 & 0.064 & 0.064 & 0.54 & 0.179 & 0.207 & 0.198 & 0.07 & $* * *$ \\
\hline 1929 & 2-phenylethanol & rosy & $\mathrm{MS}, \mathrm{A}, \mathrm{RI}$ & 0.433 & 0.034 & 0.155 & 0.99 & 0.158 & 0.155 & 0.141 & 0.06 & n.s. \\
\hline 2030 & 2-methoxy-4-ethylphenol & & MS, RIL ${ }^{a}$ & 0.051 & 0.037 & 0.034 & 0.23 & 0.052 & 0.066 & 0.053 & 0.13 & $* * *$ \\
\hline 2117 & $\begin{array}{l}\text { 1-(2,3,6-trimethylphenyl)-3-buten- } \\
\text { 2-one } \mathrm{e}^{\mathrm{T}}\end{array}$ & & MS & 0.072 & 0.112 & 0.136 & 0.30 & 0.139 & 0.291 & 0.266 & 0.35 & $* * *$ \\
\hline 2183 & 4-ethylphenol & leather & $\mathrm{MS}, \mathrm{A}, \mathrm{RIL}^{\mathrm{a}}$ & 0.045 & 0.067 & n.i. & 0.28 & 0.176 & 0.205 & 0.168 & 0.11 & $* * *$ \\
\hline 2445 & benzoic acid & fruity & $\mathrm{MS}, \mathrm{A}, \mathrm{RIL}^{\mathrm{a}}$ & n.i. & 0.016 & 0.010 & 0.28 & n.i. & 0.025 & n.i. & --- & $* * *$ \\
\hline 2512 & $\begin{array}{l}\text { 3,5-di-tert-butyl-4- } \\
\text { hydroxybenzaldehyde }\end{array}$ & & MS & 0.018 & 0.017 & 0.017 & 0.04 & 0.013 & 0.046 & n.i. & 0.79 & $* * *$ \\
\hline
\end{tabular}


TABLE 1 (CONTINUED)

\begin{tabular}{|c|c|c|c|c|c|c|c|c|c|c|c|c|}
\hline \multirow[t]{2}{*}{ RI } & \multirow[t]{2}{*}{ Compound $^{1}$} & \multirow[t]{2}{*}{ Descriptor } & \multirow[t]{2}{*}{ Identification $^{2}$} & \multicolumn{3}{|c|}{$\begin{array}{c}\text { Mean concentration } \\
(\mathrm{mg} / \mathrm{L})^{3} \\
\end{array}$} & \multirow[t]{2}{*}{ CV } & \multicolumn{3}{|c|}{$\begin{array}{c}\text { Mean concentration } \\
(\mathrm{mg} / \mathrm{L})\end{array}$} & \multirow[t]{2}{*}{ CV } & \multirow[t]{2}{*}{ Judge $^{4}$} \\
\hline & & & & CXO1 & $\mathrm{CXO3}$ & $\mathrm{CXO2}$ & & HXO1 & HXO2 & HXO3 & & \\
\hline 2540 & diisopropyl phthalate ${ }^{\mathrm{T}}$ & plastic & MS,A & 0.278 & 0.660 & 0.164 & 0.71 & 0.158 & 0.129 & 0.136 & 0.11 & $*$ \\
\hline 2549 & vanillin & vanilla & MS,A,RI & 0.008 & 0.010 & 0.012 & 0.20 & n.i. & 0.061 & 0.058 & 0.04 & $* * *$ \\
\hline \multirow[t]{3}{*}{2906} & dibutyl phthalate & plastic & MS,A,RIL ${ }^{c}$ & 0.589 & 0.142 & 0.129 & 0.92 & 0.223 & 0.218 & 0.215 & 0.02 & n.s. \\
\hline & Total & & & 1.704 & 1.336 & 0.938 & 0.29 & 1.433 & 1.848 & 1.620 & 0.13 & n.s. \\
\hline & Terpenes and norisoprenoids & & & & & & & & & & & \\
\hline 1422 & (E)-linalool oxide & floral & MS,A,RIL ${ }^{\mathrm{b}}$ & 0.021 & 0.020 & 0.035 & 0.34 & 0.054 & 0.064 & 0.069 & 0.12 & $* * *$ \\
\hline 1462 & (Z)-linalool oxide & woody, floral & MS,A,RI & 0.021 & 0.018 & 0.020 & 0.07 & 0.030 & 0.031 & 0.031 & 0.03 & $* * *$ \\
\hline 1506 & nerol & floral, sweet & MS,A,RI & 0.026 & 0.012 & 0.017 & 0.40 & 0.027 & 0.033 & 0.029 & 0.09 & $* * *$ \\
\hline 1689 & $\alpha$-terpineol & & MS, RIL ${ }^{a}$ & 0.114 & 0.105 & n.i. & 0.06 & 0.197 & 0.207 & 0.245 & 0.12 & $* * *$ \\
\hline 1742 & $\begin{array}{l}\text { 1,1,6-trimethyl-1,2-dihydrona } \\
\text { phthalene }\end{array}$ & asphalt & MS,A,RI & 0.231 & 0.265 & 0.353 & 0.22 & 0.043 & 0.046 & 0.060 & 0.18 & $* * *$ \\
\hline 1763 & $\beta$-citronellol & tea, spicy & MS,A,RI & 0.066 & 0.035 & 0.045 & 0.32 & 0.026 & 0.070 & 0.097 & 0.56 & $* * *$ \\
\hline 1818 & $\beta$-damascenone & floral, sweet & MS,A,RI & 0.093 & 0.108 & 0.110 & 0.09 & 0.331 & 0.299 & 0.276 & 0.09 & $* * *$ \\
\hline 1856 & (E)-geranyl acetone & & MS,RIL ${ }^{c}$ & 0.042 & 0.054 & 0.052 & 0.13 & 0.097 & 0.118 & 0.079 & 0.20 & $* * *$ \\
\hline 2037 & nerolidol & floral & MS, RIL ${ }^{c}$ & 0.105 & 0.161 & 0.157 & 0.22 & 0.363 & 0.340 & 0.256 & 0.18 & $* * *$ \\
\hline 2197 & cadinol $^{\mathrm{T}}$ & & MS & 0.004 & 0.017 & n.i. & 0.88 & 0.007 & 0.037 & n.i. & 0.99 & $* * *$ \\
\hline \multirow[t]{3}{*}{2361} & farnesol & floral & MS,A,RI & 0.012 & 0.010 & 0.012 & 0.07 & 0.018 & 0.013 & 0.017 & 0.17 & $* * *$ \\
\hline & Total & & & 0.735 & 0.805 & 0.801 & 0.15 & 1.193 & 1.258 & 1.159 & 0.05 & $* * *$ \\
\hline & Aldehydes and ketones & & & & & & & & & & & n.s. \\
\hline 10 & acetaldehyde & fruity & MS,A,RI & 0.049 & 0.031 & 0.037 & 0.22 & 0.039 & n.i. & n.i. & --- & n.s. \\
\hline 1082 & hexanal & green & MS,A,RI & n.i. & n.i. & 0.001 & --- & n.i. & n.i. & 0.007 & --- & n.s. \\
\hline 1195 & heptan-2-one & floral, green & $\mathrm{MS}, \mathrm{A}, \mathrm{RIL}^{\mathrm{b}}$ & n.i. & 0.007 & 0.004 & 0.35 & n.i. & 0.007 & 0.005 & 0.16 & $* * *$ \\
\hline 1482 & decanal & & MS,RI & 0.033 & 0.018 & 0.016 & 0.41 & n.i. & 0.102 & 0.058 & 0.39 & $* * *$ \\
\hline \multirow[t]{3}{*}{1598} & undecan-2-one & & $\mathrm{MS}, \mathrm{RIL}^{\mathrm{a}}$ & n.i. & 0.007 & 0.007 & 0.00 & 0.014 & 0.018 & 0.017 & 0.12 & $* * *$ \\
\hline & Total & & & 0.081 & 0.063 & 0.064 & 0.15 & 0.054 & 0.127 & 0.087 & 0.41 & $* * *$ \\
\hline & Furans & & & & & & & & & & & \\
\hline 1452 & furfural & toasty & MS,A,RI & 0.577 & 0.584 & 0.602 & 0.02 & 1.458 & 1.120 & 1.697 & 0.20 & $* * *$ \\
\hline 1571 & 5-methylfurfural & roasted & MS,A,RI & 0.050 & 0.049 & 0.045 & 0.06 & 0.095 & 0.115 & 0.079 & 0.19 & $* * *$ \\
\hline 1622 & ethyl 2-furoate & balsamic & MS,A,RIL ${ }^{b}$ & 0.007 & 0.055 & 0.059 & 0.73 & 0.147 & 0.337 & 0.419 & 0.46 & $* * *$ \\
\hline \multirow[t]{3}{*}{2093} & $\begin{array}{l}\text { 2,5-dihydro-2,2-dimethyl-5-(1- } \\
\text { methylethenyl)-3-(1-methylethyl)- }_{\text {furan }^{\mathrm{T}}}\end{array}$ & & MS & 0.211 & 0.105 & 0.177 & 0.33 & 0.071 & 0.156 & 0.147 & 0.38 & $* *$ \\
\hline & Total & & & 0.845 & 0.792 & 0.883 & 0.05 & 1.770 & 1.729 & 2.341 & 0.18 & $* *$ \\
\hline & Lactones & & & & & & & & & & & \\
\hline 1888 & $\delta$-nonalactone ${ }^{\mathrm{T}}$ & & MS & 0.025 & 0.037 & 0.038 & 0.22 & 0.025 & 0.037 & 0.038 & 0.22 & n.s. \\
\hline 1961 & $\gamma$-nonalactone & cream, coconut & MS,A,RI & 0.003 & 0.010 & n.i. & 0.85 & 0.074 & 0.075 & 0.089 & 0.11 & $* * *$ \\
\hline \multirow[t]{3}{*}{2120} & $\gamma$-decalactone & & MS, RIL ${ }^{\mathrm{d}}$ & 0.007 & 0.007 & 0.007 & 0.00 & 0.010 & 0.024 & 0.029 & 0.45 & $* * *$ \\
\hline & Total & & & 0.034 & 0.054 & 0.045 & 0.22 & 0.110 & 0.135 & 0.156 & 0.17 & $* * *$ \\
\hline & Acetals & & & & & & & & & & & \\
\hline \multirow[t]{2}{*}{1294} & 1,1,3-triethoxypropane & fruity, vegetal & $\mathrm{MS}, \mathrm{A}, \mathrm{RIL}^{\mathrm{d}}$ & 0.013 & 0.007 & 0.010 & 0.33 & n.i. & 0.008 & 0.007 & 0.13 & $* * *$ \\
\hline & Total & & & 0.013 & 0.007 & 0.010 & 0.33 & n.i. & 0.008 & 0.007 & 0.13 & $* * *$ \\
\hline
\end{tabular}

RI: Retention index

${ }^{1}$ Tentatively identified by mass spectra

${ }^{2}$ Identified by MS (mass spectra), A (aroma descriptors), RI (retention index), and RIL (retention indices from literature).

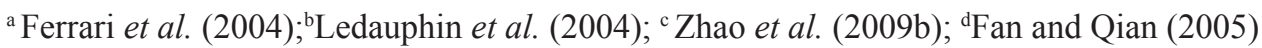

${ }^{3}$ n.i.: Not identified; CV: Coefficient of variation of concentrations in three Changyu and Hennessy XO samples

${ }^{4}$ Judge: Difference between mean concentration of three Changyu samples and that of three Hennessy samples

$*$ Difference at $0.05 ; * *$ Difference at $0.01 ; * * *$ Difference at $0.001 ;{ }^{\text {n.s }}$ No significant difference 
most of which have already been identified by other authors (Ferrari et al., 2004; Ledauphin et al., 2004; Janacova et al., 2008). Among these volatiles, 118 compounds were found to be common to both Changyu XO and Hennessy XO, and 21 and 36 volatiles were specific to Changyu XO and Hennessy XO, respectively.

\section{GC-O analysis}

The aroma compounds obtained in the six brandies by GC-O are shown in Tables 1,2 and 3. A total of 92 aroma compounds were identified in the six brandies. The most abundant perceived aromas were descriptors such as fruity, floral, alcohol, grass and green, and rancid, for Changyu XO samples. Besides grass and green, the majority of these descriptors have been found in three Hennessy XO samples. Among the 92 aroma compounds, 71 aromas were common to both Changyu XO and Hennessy $\mathrm{XO}$, and nine and twelve compounds were specific to Changyu XO and Hennessy XO, respectively.

\section{Comparison of volatile compounds in Changyu and Hennessy XO \\ Esters}

Esters were the most abundant volatile compounds in both Changyu XO and Hennessy XO, with ethyl esters dominating this class. As seen in Table 1, 47 esters were common compounds, and the whole average concentration (abbreviated to $\left.\mathrm{AC}_{\mathrm{w}}\right)$ of esters in Changyu $\mathrm{XO}(57.836 \mathrm{mg} / \mathrm{L})$ was lower than that in Hennessy XO $(63.438 \mathrm{mg} / \mathrm{L})$. According to the T-test, no significant difference was found in $\mathrm{AC}_{\mathrm{w}}$ of esters in Changyu XO and Hennessy XO. However, there were significant differences in average concentrations (abbreviated to AC) of each ester, excepting 3-methylbutyl acetate, ethyl octanoate, 3-methylbutyl 2-hydroxypropanoate, diethyl pentanedioate, ethyl 2,3-diethoxypropanoate, and ethyl dodecanoate. Three esters, i.e. ethyl octanoate, ethyl decanoate and ethyl dodecanoate, were the most concentrated compounds and covered up to $80 \%$ of the whole of the ester concentrations in both Changyu XO and Hennessy XO. These esters were the most important skeleton compounds in the brandy samples and revealed a low coefficient of variation $(\mathrm{CV} \leq 20 \%)$.

Esters are mostly formed through the esterification of alcohols with fatty acids during fermentation, distillation and the ageing processes (Ledauphin et al., 2003; Fan and Qian, 2005; Zhao et al., 2009a). Of these volatile esters, 22 aroma compounds were identified by GC-O. Esters mainly contribute fruity, floral, pineapple, apple-like and banana-like aromas (Fan and Qian, 2006). For example, ethyl butanoate, ethyl heptanoate and ethyl 2-hydroxypropanoate generated fruity aroma; pineapple aroma was explained by the presence of ethyl acetate; ethyl 3-methylbutanoate was responsible for apple aroma; and ethyl 2-hydroxy-3-methylbutanoate imparted floral aromas. According to statistical analysis, there were significant differences in these aroma esters in both Changyu XO and Hennessy XO, except for 3-methylbutyl acetate and ethyl octanoate.

As seen in Table 1, ethyl pentanoate, isopentyl isopentanoate and 2-ethylhexyl acetate were specific to Changyu XO. Of these, ethyl pentanoate and isopentyl isopentanoate imparted apple and fruity odours to the global aroma. Table 2 shows that eight esters, including ethyl hex-2-enoate, 2-methylpropyl acetate, isobutyl hexanoate, propyl octanoate, isobutyl octanoate, methyl dodecanoate, isobutyl dodecanoate, and decyl decanoate, were specific to Hennessy XO, and 2-Methylpropyl acetate, ethyl hex-2-enoate, isobutyl hexanoate, propyl octanoate, and isobutyl octanoate were detected with floral and fruity odours.

\section{Alcohols}

Alcohols formed the second group of concentrated compounds in these samples. As seen in Table 1, 21 alcohols were common to both Changyu XO and Hennessy XO. Similar to esters, the $\mathrm{AC}_{\mathrm{w}}$ of alcohols in Changyu XO $(19.728 \mathrm{mg} / \mathrm{L})$ were lower than those in Hennessy XO $(29.168 \mathrm{mg} / \mathrm{L})$. According to the T-test, a significant difference between the $\mathrm{AC}_{\mathrm{W}}$ of alcohols in Changyu $\mathrm{XO}$ and Hennessy XO was identified. Moreover, significant differences were also found in the AC of most alcohols, excepting 2-methylpropanol, 3-ethoxypropanol, heptan-1-ol and tetradecan-1-ol. The most concentrated of the compounds, covering up to $80 \%$ of the whole alcohol concentrations in both Changyu XO and Hennessy XO, was 3-methylbutanol. The $\mathrm{CV}$ levels of 3-methylbutanol in Changyu XO and Hennessy XO were $1 \%$ and $2 \%$, respectively. Based on these results, 3-methylbutanol was the most important skeleton compound in the brandies. Four alcohols, i.e., 2,3-butanediol, nonan2-ol, undecan-2-ol and 2-tetradecen-1-ol, were only found in Hennessy XO. Butan-2-ol was specific to Changyu XO.

Among these alcohols, 13 were identified as aroma compounds. Most alcohols have high sensory thresholds and impart fruity, fusel, floral, grass, and alcohol-like aromas (Fan and Qian, 2006). Propan-1-ol and butan-1-ol generated alcohol and fruity odours; fusel aroma was explained by the presence of 2-methylpropanol and 3-methylbutanol; (E)-hex-3en-1-ol and (Z)-hex-3-en-1-ol were responsible for grass, leaf and green aromas; decan-1-ol contributed to fatty aroma and dodecan-1-ol imparted a rancid aroma. Significant differences in the concentrations of the 12 aroma alcohols, except for 2-methylpropanol, were recorded (Table 1).

\section{Acids}

Acids are mainly derived from the grapes. Small amounts of acids were formed from amino acids catalysed by yeast under anaerobic conditions (Watts et al., 2003). In the current analysis, a total of 18 acids were identified in Changyu XO and Hennessy XO. Eleven of these acids were common to both brands, whereas five acids, including isobutanoic, 4-methylhexanoic, 2-ethyl hexanoic, 3-ethylhepatanoic, and tridecanoic acid, were specific to Hennessy XO. Two acids involving hexanoic and heptanoic acid were found specific to Changyu XO. The $\mathrm{AC}_{\mathrm{w}}$ of acids in Changyu XO $(22.222 \mathrm{mg} / \mathrm{L})$ was lower than in Hennessy XO $(26.978 \mathrm{mg} / \mathrm{L})$. According to the T test, there was a significant difference in the total concentration of acids between Changyu XO and Hennessy XO. Moreover, there were significant differences in the AC of most acids, except for butanoic acid, octanoic acid and pentadecanoic acid. Decanoic acid and octanoic acid were the most important acid compounds, comprising up to $90 \%$ of the total acid concentrations in both Changyu XO and Hennessy XO. Of these acids, six aroma-active acids, including acetic acid, butanoic acid, 2/3-methylbutanoic acid, octanoic acid, nonanoic acid and decanoic acid, were identified by GC-O. These acids mainly contribute to rancid and vinegar odours for the global aroma of both brand brandies. 


\section{Benzene derivatives}

Benzene derivatives were identified as the fourth largest volatile group in the brandies, followed by esters, alcohols and acids. The $\mathrm{AC}_{\mathrm{W}}$ of benzene derivatives in Changyu XO (1.326 $\mathrm{mg} / \mathrm{L})$ was slightly lower than those in Hennessy XO (1.634 $\mathrm{mg} / \mathrm{L})$. No significant differences between Changyu XO and Hennessy XOwere observed in the $\mathrm{AC}_{\mathrm{W}}$ of benzene derivatives by T-test analysis. Fifteen benzene derivatives were common to both Changyu XO and Hennessy XO. In Changyu XO samples, 2-phenylethanol, 1-(2,3,6-trimethylphenyl)-3-buten-2-one, diisopropyl phthalate, and dibutyl phthalate were the higher concentrated compounds. Six compounds were specific to Changyu XO, including benzaldehyde, butyl benzoate, eugenol, 2,4-(1,1-dimethylethyl) phenol, 2-phenylethyl octanoate, and benyl benzoate. As for Hennessy XO, the number of benzene derivatives were less than that contained in Changyu $\mathrm{XO}$; and ethyl benzenepropanoate, 1-(2,3,6-trimethylphenyl)3-buten-2-one, 4-ethylphenol, and dibutyl phthalate were the important compounds. Of these, dibutyl phthalate, 4-ethylphenol and ethyl benzenepropanoate (CV $\leq 20 \%)$ were considered as the skeleton compounds in Hennessy XO.

Among these benzene derivatives, 11 aroma compounds were identified by GC-O. Ethyl benzoate, 2-phenylethyl acetate, benzyl alcohol and ethyl benzenepropanoate contributed to floral aromas; the honey aroma was explained by the presence of ethyl phenylacetate; 2-phenylethanol imparts a rosy aroma; and vanillin aromas are explained by vanillin. 4-Ethylphenol, with an undesirable leather odour, was produced by the contaminant yeasts Brettanomyces/Dekkera from grape-derived phenolic acids (Bautista-ortín et al., 2008; Garde-Cerdan \& AncinAzpilicueta, 2006; Martorell et al., 2002). Plastic aroma, as an off-flavour, mainly explained by diisopropyl phthalate and dibutyl phthalate, has been identified by Zhao et al. (2009b). This aroma in brandy may be introduced during wine-making through exposing wine to plastic equipment.

\section{Terpenes and norisoprenoids}

Compared to the volatile compounds discussed above, all other volatiles, including terpenes, norisoprenoids, aldehydes, ketones, furans, lactones and acetals, had relatively lower concentrations and lower numbers, but they also played an important role in the development of brandy flavour due to their special and unique characteristics.

A total of twelve terpenes and three norisoprenoids were detected in Changyu XO and Hennessy XO. Among them, three compounds, namely linalool, geraniol and $\beta$-ionone, were specific to Changyu $\mathrm{XO}$, whereas $\gamma$-terpineol was uniquely detected in Hennessy XO. The $\mathrm{AC}_{\mathrm{W}}$ of terpenes in Changyu XO $(0.780 \mathrm{mg} / \mathrm{L})$ was nearly half of that in Hennessy XO $(1.203$ $\mathrm{mg} / \mathrm{L}$ ). According to the T-test, significant difference was found in the $\mathrm{AC}$ of all the terpenes. Of these, nerolidol, $\alpha$-terpineol and $\beta$-damascenone occurred in a comparatively higher concentration than the other compounds in both Changyu XO and Hennessy XO.

Terpenes largely originate from grapes (Ferrari et al., 2004). $\beta$-Damascenone, a sweet odorant, mainly comes from the degradation of carotenoids in grapes (Strauss et al., 1987; Buttery et al., 1990). Though present in a low content, the terpenes and norisoprenoids were important due to their low aroma threshold values. Among these volatiles, a total of 10 terpenes and norisoprenoids were identified by GC-O; these were (E)-linalool oxide and farnesol (floral aroma), (Z)linalool oxide (woody, floral aroma), nerol (floral aroma), $\beta$-damascenone (sweet aroma), and $\beta$-citronellol (tea, spicy odour). Geraniol and $\beta$-ionone, with sweet, floral odour, were specific to Changyu XO samples, whereas, $\gamma$-terpineol gave a lime odour to Hennessy XO samples. The 1,1,6-trimethyl-1,2dihydronaphthalene (TDN) content was lower in Hennessy $\mathrm{XO}$ than in Changyu XO; it imparts asphalt tones and has been reported as an off-flavour in wine.

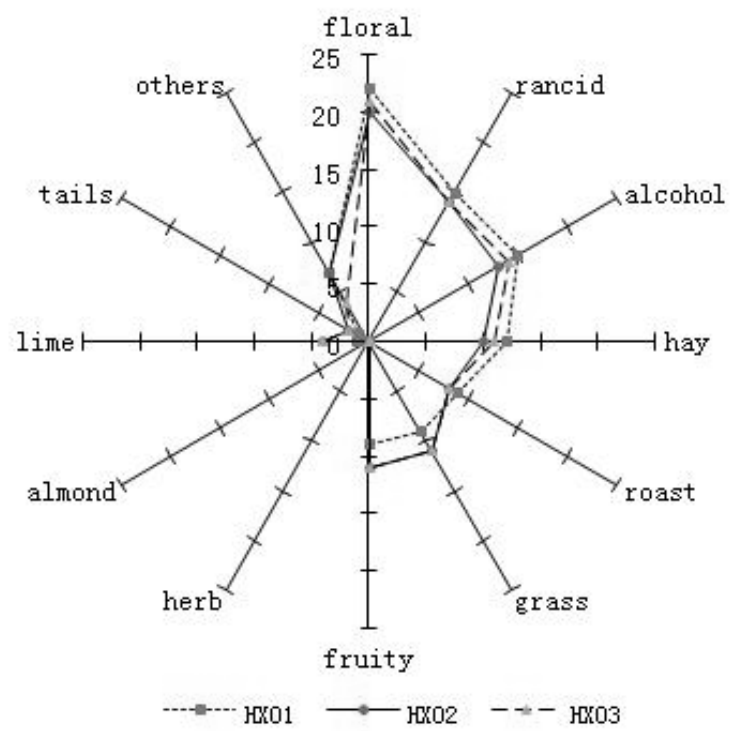

FIGURE 1

The aroma profiles obtained for Hennessy XO and Changyu XO. 

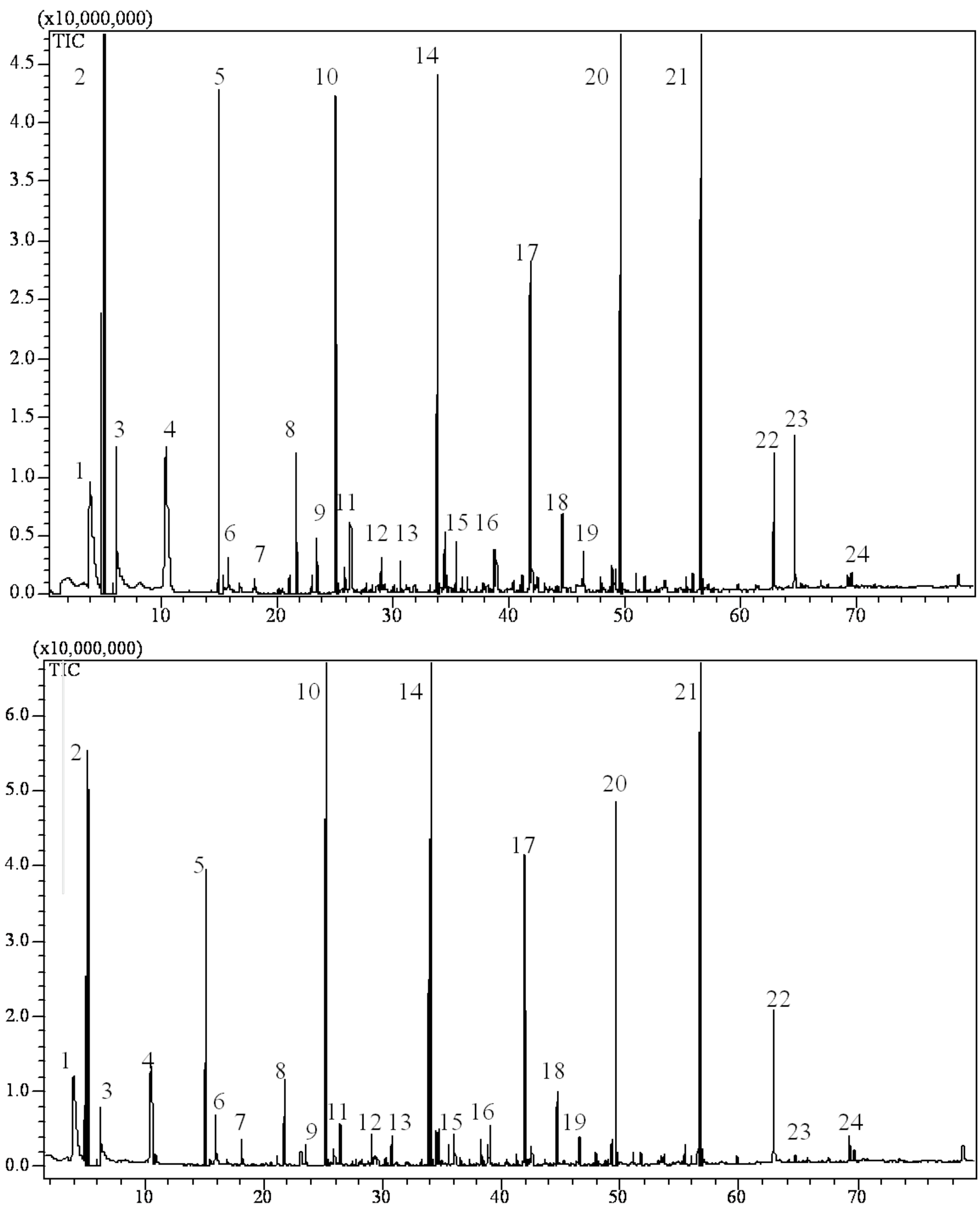

FIGURE 2

GC-MS chromatograms obtained for Changyu XO1 (a) and Hennessy XO1 (b).

Note: 1, ethyl acetate; 2, ethanol; 3, ethyl butanoate; 4, 2-methylpropanol; 5, 3-methylbutanol; 6, ethyl hexanoate; 7, ethyl orthoformate; 8, hexan-1-ol; 9, octan-3-ol; 10, ethyl octanoate; 11, furfural; 12, ethyl nonanoate; 13, octan-1-ol; 14, ethyl decanoate; 15, diethyl succinate; 16, decan-1-ol; 17, ethyl dodecanoate; 18, 2-phenylethanol; 19, dodecan-1-ol; 20, octanoic acid; 21, decanoic acid; 22, dodecanoic acid; 23, diisopropyl phthalate; 24, dibutyl phthalate. 
TABLE 2

Special volatile compounds in Changyu XO samples by GC-MS on a DB-Wax column.

\begin{tabular}{|c|c|c|c|c|c|c|c|}
\hline \multirow[t]{2}{*}{ RI } & \multirow[t]{2}{*}{ Compound $^{1}$} & \multirow[t]{2}{*}{ Descriptor } & \multirow[t]{2}{*}{ Identification $^{2}$} & \multicolumn{3}{|c|}{$\begin{array}{c}\text { Mean concentration } \\
(\mathrm{mg} / \mathrm{L})^{3}\end{array}$} & \multirow[t]{2}{*}{$\mathbf{C V}^{4}$} \\
\hline & & & & CXO1 & CXO2 & CXO3 & \\
\hline & Esters & & & & & & \\
\hline 1137 & ethyl pentanoate & apple & MS,A,RI & 0.016 & n.i. & n.i. & --- \\
\hline 1275 & isopentyl isopentanoate & fruity & MS,A,RIL ${ }^{b}$ & 0.043 & 0.005 & 0.046 & 0.730 \\
\hline \multirow[t]{3}{*}{1374} & 2-ethylhexyl acetate & & MS, RIL $^{b}$ & n.i. & 0.003 & n.i. & --- \\
\hline & Total & & & 0.059 & 0.008 & 0.046 & 0.70 \\
\hline & Alcohols & & & & & & \\
\hline \multirow[t]{3}{*}{1016} & butan-2-ol & & MS, RIL $^{\mathrm{b}}$ & 0.002 & n.i. & n.i. & --- \\
\hline & Total & & & 0.002 & n.i. & n.i. & --- \\
\hline & Acids & & & & & & \\
\hline 1849 & hexanoic acid & & MS,RI & n.i. & 0.126 & 0.131 & 0.030 \\
\hline \multirow[t]{3}{*}{1951} & heptanoic acid & & MS,RI & 0.030 & 0.036 & 0.023 & 0.220 \\
\hline & Total & & & 0.030 & 0.162 & 0.154 & 0.640 \\
\hline & Benzenes and derivatives & & & & & & \\
\hline 1510 & benzaldehyde & almond & MS,A,RI & 0.132 & 0.185 & n.i. & 0.240 \\
\hline 1788 & butyl benzoate ${ }^{\mathrm{T}}$ & & MS & n.i. & 0.017 & n.i. & --- \\
\hline 2156 & eugenol & & MS,RI & 0.011 & n.i. & 0.010 & 0.070 \\
\hline 2351 & 2,4-(1,1-dimethylethyl)phenol ${ }^{\mathrm{T}}$ & herb & MS,A & 0.016 & 0.014 & 0.017 & 0.100 \\
\hline 2377 & 2-phenylethyl octanoate ${ }^{\mathrm{T}}$ & & MS & n.i. & 0.008 & 0.006 & 0.200 \\
\hline \multirow[t]{3}{*}{2639} & benzyl benzoate & & MS, RIL ${ }^{\mathrm{c}}$ & 0.026 & 0.015 & 0.022 & 0.270 \\
\hline & Total & & & 0.185 & 0.239 & 0.055 & 0.59 \\
\hline & Terpenes and norisoprenoids & & & & & & \\
\hline 1555 & linalool & & MS,RI & 0.111 & n.i. & 0.067 & 0.350 \\
\hline 1851 & Geraniol & sweet, rosy & MS,A,RI & n.i. & 0.018 & 0.026 & 0.260 \\
\hline \multirow[t]{3}{*}{1924} & $\beta$-ionone & floral & MS,A,RI & 0.035 & 0.043 & 0.046 & 0.140 \\
\hline & Total & & & 0.146 & 0.061 & 0.139 & 0.41 \\
\hline & Aldehydes and ketones & & & & & & \\
\hline 1202 & 3-ethoxypropanal & fusel & $\mathrm{MS}, \mathrm{A}, \mathrm{RIL}^{\mathrm{b}}$ & 0.007 & 0.007 & n.i. & 0.000 \\
\hline \multirow[t]{3}{*}{1235} & octan-3-one & & MS, RIL $^{\mathrm{b}}$ & 0.008 & 0.004 & 0.006 & 0.330 \\
\hline & Total & & & 0.015 & 0.011 & 0.006 & 0.420 \\
\hline & Acetals & & & & & & \\
\hline \multirow[t]{2}{*}{978} & 1,1-diethoxy-2-methylpropane & & MS, RIL ${ }^{d}$ & 0.003 & 0.003 & n.i. & 0.000 \\
\hline & Total & & & 0.003 & 0.003 & n.i. & 0.000 \\
\hline
\end{tabular}

RI: Retention index.

${ }^{1}$ Tentatively identified by mass spectra

2 Identified by MS (mass spectra), A (aroma descriptors), RI (retention index), and RIL (retention indices from literature).

${ }^{\mathrm{a}}$ Ferrari et al. (2004); ${ }^{\mathrm{b}}$ Ledauphin et al. (2004); ${ }^{\mathrm{C}}$ Zhao et al. (2009b); ${ }^{\mathrm{d}}$ Fan \& Qian (2005)

${ }^{3}$ n.i.: Not identified.

${ }^{4} \mathrm{CV}$ : Coefficient of variation of concentrations in three Changyu and Hennessy XO samples.

\section{Aldehydes and ketones}

Only five aldehydes and ketones were common to both Changyu $\mathrm{XO}$ and Hennessy $\mathrm{XO}$. The $\mathrm{AC}_{\mathrm{W}}$ of aldehydes and ketones were $0.070 \mathrm{mg} / \mathrm{L}$ and $0.089 \mathrm{mg} / \mathrm{L}$ in Changyu XO and Hennessy XO, respectively. According to the T-test, there were no significant differences in the AC of acetaldehyde and hexanal, whereas a significant difference was found in the other three compounds; 3-ethoxypropanal and octan-3-one were specific in Changyu $\mathrm{XO}$, and nonanal and 11-dodecen-2-one were specific to Hennessy XO. Of these, five aroma compounds, acetaldehyde, hexanal, 3-ethoxypropanal, nonanal and heptan-2-one included, were identified. These compounds contributed green and fruity aromas to the global aroma of brandies.

\section{Furans}

Four furans common to both brands of brandy were identified. The $\mathrm{AC}_{\mathrm{W}}$ of furans in Changyu XO $(0.84 \mathrm{mg} / \mathrm{L})$ was markedly lower than that in Hennessy XO $(1.947 \mathrm{mg} / \mathrm{L})$. According to the T-test, there were significant differences between Changyu XO and Hennessy XO in the whole concentrations of furans and in the AC of all furans. Of these furans, furfural was the most concentrated compound, with concentrations of up to $70 \%$ of the whole concentrations of furans. Furans are primarily oak derived, but also form in the hot conditions of distillation (Van Jaarsveld et al., 2009a,b,c). Among these furans, three were aroma compounds. Toasty, roasted and balsamic aromas were explained by furfural, 5-methylfurfural and ethyl 2-furoate, respectively. 
TABLE 3: Special volatile compounds in Hennessy XO samples by GC-MS on a DB-Wax column

\begin{tabular}{|c|c|c|c|c|c|c|c|}
\hline \multirow[t]{2}{*}{$\mathbf{R I}$} & \multirow[t]{2}{*}{ Compound $^{1}$} & \multirow[t]{2}{*}{ Descriptor } & \multirow[t]{2}{*}{ Identification $^{2}$} & \multicolumn{3}{|c|}{$\begin{array}{l}\text { Mean concentration } \\
(\mathrm{mg} / \mathrm{L})^{3}\end{array}$} & \multirow[t]{2}{*}{$\mathrm{CV}^{4}$} \\
\hline & & & & HXO1 & HXO2 & HXO3 & \\
\hline & Esters & & & & & & \\
\hline 985 & 2-methylpropyl acetate & floral & MS,A,RI & n.i. & n.i. & 0.013 & --- \\
\hline 1333 & ethyl hex-2-enoate & fruity & MS,A,RIL ${ }^{a}$ & n.i. & 0.004 & 0.002 & 0.47 \\
\hline 1345 & isobutyl hexanoate $^{\mathrm{T}}$ & fruity & MS,A & n.i. & 0.003 & n.i. & --- \\
\hline 1509 & propyl octanoate $^{\mathrm{T}}$ & fruity & MS,A & 0.016 & n.i. & n.i. & --- \\
\hline 1558 & isobutyl octanoate $^{\mathrm{T}}$ & fruity & MS,A & 0.079 & n.i. & n.i. & --- \\
\hline 1801 & methyl dodecanoate ${ }^{\mathrm{T}}$ & & MS & 0.034 & 0.061 & 0.049 & 0.28 \\
\hline 1957 & isobutyl dodecanoate $^{\mathrm{T}}$ & & MS & 0.003 & 0.024 & 0.005 & 1.05 \\
\hline \multirow[t]{3}{*}{2658} & decyl decanoate $^{\mathrm{T}}$ & & MS & 0.026 & 0.017 & 0.026 & 0.23 \\
\hline & Total & & & 0.158 & 0.102 & 0.08 & 0.35 \\
\hline & Alcohols & & & & & & \\
\hline 1410 & 2,3-butanediol & & $\mathrm{MS}, \mathrm{RIL}^{\mathrm{a}}$ & n.i. & 0.01 & 0.002 & 0.94 \\
\hline 1513 & nonan-2-ol & & MS,RIL ${ }^{\mathrm{b}}$ & 0.221 & 0.305 & n.i. & 0.23 \\
\hline 1721 & undecan-2-ol & & $\mathrm{MS}, \mathrm{RIL}^{\mathrm{b}}$ & 0.041 & 0.051 & 0.053 & 0.13 \\
\hline \multirow[t]{3}{*}{2029} & 2-tetradecen-1-ol ${ }^{\mathrm{T}}$ & & MS & n.i. & n.i. & 0.002 & --- \\
\hline & Total & & & 0.262 & 0.356 & 0.055 & 0.69 \\
\hline & Acids & & & & & & \\
\hline 1563 & isobutanoic acid & rancid & MS,A,RI & 0.0180 & n.i. & n.i. & --- \\
\hline 1932 & 4-methylhexanoic acid ${ }^{\mathrm{T}}$ & & MS & n.i. & 0.023 & 0.028 & 0.14 \\
\hline 1948 & 2-ethylhexanoic acid & & MS & 0.0140 & 0.051 & 0.057 & 0.57 \\
\hline 2073 & 3-ethylheptanoic acid & & MS & 0.0180 & 0.06 & 0.06 & 0.53 \\
\hline \multirow[t]{3}{*}{2659} & 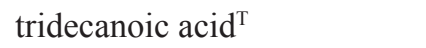 & & MS & 0.0080 & n.i. & 0.014 & 0.39 \\
\hline & Total & & & 0.058 & 0.134 & 0.159 & 0.45 \\
\hline & Terpenics & & & & & & \\
\hline \multirow[t]{3}{*}{1602} & $\gamma$-terpineol & lime & $\mathrm{MS}, \mathrm{A}, \mathrm{RI}$ & n.i. & 0.025 & n.i. & --- \\
\hline & Total & & & n.i. & 0.025 & n.i. & --- \\
\hline & Aldehydes and ketones & & & & & & \\
\hline 1384 & nonanal & fruity & MS,A,RI & n.i. & 0.017 & 0.030 & 0.400 \\
\hline \multirow[t]{3}{*}{1802} & 11-dodecen-2-one ${ }^{\mathrm{T}}$ & & MS & 0.009 & n.i. & 0.005 & 0.400 \\
\hline & Total & & & 0.009 & 0.017 & 0.035 & 0.800 \\
\hline & Acetals & & & & & & \\
\hline 885 & 1,1-diethoxyethane & fruity & MS,RIL ${ }^{\mathrm{d}}$ & 0.082 & n.i. & 0.096 & 0.120 \\
\hline 1084 & 1,1-diethoxy-2-methylbutane & & MS,A,RIL ${ }^{\mathrm{d}}$ & n.i. & 0.006 & n.i. & --- \\
\hline \multirow[t]{3}{*}{1237} & 1,1-diethoxyhexane & floral & MS,A,RIL ${ }^{\mathrm{d}}$ & 0.010 & 0.029 & n.i. & 0.670 \\
\hline & Total & & & 0.092 & 0.035 & 0.696 & 1.340 \\
\hline & Others & & & & & & \\
\hline \multirow[t]{2}{*}{1686} & 3-ethoxy-p-menth-1-en-8-ol ${ }^{\mathrm{T}}$ & & MS & 0.012 & 0.020 & 0.040 & 0.60 \\
\hline & Total & & & 0.012 & 0.020 & 0.040 & 0.60 \\
\hline
\end{tabular}

RI: Retention index

${ }^{1}$ Tentatively identified by mass spectra

${ }^{2}$ Identified by MS (mass spectra), A (aroma descriptors), RI (retention index), and RIL (retention indices from literature).

${ }^{\mathrm{a}}$ Ferrari et al. (2004); ${ }^{\mathrm{b}}$ Ledauphin et al. (2004); ${ }^{\mathrm{c}}$ Zhao et al. (2009b); ${ }^{\mathrm{d}} \mathrm{Fan}$ \& Qian (2005)

${ }^{3}$ n.i.: Not identified.

${ }^{4} \mathrm{CV}$ : Coefficient of variation of concentrations in three Changyu and Hennessy XO samples. 


\section{Lactones}

Three lactones, including $\delta$-nonalactone, $\gamma$-nonalactone and $\gamma$-decalactone, were identified as compounds common to both Changyu $\mathrm{XO}$ and Hennessy $\mathrm{XO} . \mathrm{AC}_{\mathrm{W}}$ of lactones in Changyu $\mathrm{XO}(0.044 \mathrm{mg} / \mathrm{L})$ was clearly lower than these in Hennessy XO $(0.134 \mathrm{mg} / \mathrm{L})$. Lactones are mostly derived from oak barrels, and the condition of barrels (wood type, manufacturing, prior use, etc.) greatly influence the extraction of lactones (Caldeira et al., 2002; Watts et al., 2003). In these lactones, only $\gamma$-nonalactone, with cream and coconut aromas, was identified by GC-O. According to the $\mathrm{T}$ test, the concentration of $\gamma$-nonalactone showed a significant difference between Changyu XO and Hennessy XO.

\section{Acetals}

Acetals are largely formed from the condensation of aldehydes with alcohols (Wondra and Berovic, 2001; Zhao et al., 2009a). While 1,1,3-triethoxypropane, with a fruity and vegetal aroma, was found as a unique acetal compound common to both Changyu XO and Hennessy XO, 1,1-diethoxymethane, 1,1-diethoxy-2-methylbutane, and 1,1-diethoxyhexane were specific to Hennessy XO and 1,1-diethoxy-2-methylpropane was specific to Changyu XO.

\section{CONCLUSIONS}

The current study compared the differences related to volatile compounds in Changyu XO and Hennessy XO. Three different batches of brandy selected from each brand were analysed by GC-MS coupled with HS-SPME, GC-O and sensory evaluation. A total of 160 volatile compounds were identified in the two brand samples. Among these volatiles, 85 aroma compounds responsible for brandy flavour were identified by $\mathrm{GC}-\mathrm{O}$, of which, 68 were found common to both brandies, and seven and ten were separately specific to Changyu XO and Hennessy XO. Most volatile compounds in Changyu XO had lower concentrations than those in Hennessy XO. This could be ascribed to the development of knowledge of the aroma compositions of both brandies. Judging from the results of statistical and sensory analyses, the differences found between Changyu XO and Hennessy XO are significant.

\section{LITERATURE CITED}

Bautista-ortín, A.B., Lencina, A.G., Cano-lópez, M., Pardo-mínguez, F., Lópezroca, J.M. \& Gómez-plaza, E., 2008. The use of oak chips during the ageing of a red wine in stainless steel tanks or used barrels: Effect of the contact time and size of the oak chips on aroma compounds. Aust. J. Grape Wine Res. 14, 63-70

Buttery, R.G., Teranishi, R., Ling, L.C. \& Turnbaugh, J.G.J., 1990. Quantitative induced by heat treatment. J. Food Eng. 76, 202-211.

Caldeira, I., Belchior, A.P., Climaco, M.C. \& De Sousa, R.B., 2002. Aroma profile of Portuguese brandies aged in chestnut and oak woods. Anal. Chim. Acta $458,55-62$

Caldeira, I., Climaco, M.C., De Sousa, R.B. \& Belchior, A.P., 2006. Volatile composition of oak and chestnut woods used in brandy ageing: Modification induced by heat treatment. J. Food Eng. 76, 202-211.

Fan, W.L. \& Qian, M.C., 2005. Headspace solid phase microextraction and gas chromatography-olfactometry dilution analysis of young and aged Chinese "Yanghe Daqu" liquors. J. Agr. Food Chem. 53, 7931-7938.
Fan, W.L. \& Qian, M.C., 2006. Characterization of aroma compounds of Chinese "Wuliangye" and "Jiannanchun" liquors by aroma extract dilution analysis. J. Agr. Food Chem. 54, 2695-2704.

Ferrari, G., Lablanquie, O., Cantagrel, R., Ledauphin, H., Payot, T., Fournier, N. \& Guichard, E., 2004. Determination of key odorant compounds in freshly distilled Cognac using GC-O, GC-MS, and sensory evaluation. J. Agr. Food Chem. 52, 5670-5676.

Garde-Cerdan, T. \& Ancin-Azpilicueta, C., 2006. Review of quality factors on wine ageing in oak barrels. Trends Food Sci. Technol. 17, 438-447.

Go'mez-M1'guez, M.J., Go'mez-M1'guez, M., Vicario, I.M. \& Heredia, F.J., 2007. Assessment of colour and aroma in white wines vinifications: Effects of grape maturity and soil type. J. Food Eng. 79, 758-764.

Howard, K.L., Mike, J.H. \& Riesen, R., 2005. Validation of a solid-phase microextraction method for headspace analysis of wine aroma components. Am. J. Enol. Vitic. 56, 37-45.

Janacova, A., Sadecka, J., Kohajdova, Z. \& Spanik, I., 2008. The identification of aroma-active compounds in Slovak brandies using GC-sniffing, GC-MS and sensory evaluation. Chromatographia 67, 113-121.

Ledauphin, J., Guichard, H., Saint-Clair, J.F., Picoche, B. \& Barillier, D., 2003. Chemical and sensorial aroma characterization of freshly distilled Calvados. 2. Identification of volatile compounds and key odorants. J. Agr. Food Chem. $51,433-442$.

Ledauphin, J., Saint-Clair, J.F., Lablanquie, O., Guichard, H., Founier, N., Guichard, E. \& Barillier, D., 2004. Identification of trace volatile compounds in freshly distilled calvados and cognac using preparative separations coupled with gas chromatography-mass spectrometry. J. Agr. Food Chem. 52, 51245134

Martorell, N., Marti, M.P., Mestres, M., Busto, O. \& Guasch, J., 2002. Determination of 4-ethylguaiacol and 4-ethylphenol in red wines using headspace-solid-phase microextraction-gas chromatography. J. Chromatogr. A 95, 349-354.

Milicevic, B., Banovic, M., Kovacevic-Ganic, K. \& Gracin, L., 2002. Impact of grape varieties on wine distillates flavour. Food Technol. Biotechnol. 40, 227-232.

Onishi, M., Crowell, E.A. \& Guymon, J.F., 1978. Comparative Composition of brandies from Thompson Seedless and three white-wine grape varieties. Am. J. Enol. Vitic. 29, 54-59.

Pérez-Coello, M.S., Sanz, J. \& Cabezudo, M.D., 1995. Gas chromatographicmass spectrometric analysis of volatile compounds in oak wood used for ageing of wines and spirits. Chromatographia 47, 427-432.

Steel, R.G.D. \& Torrie, J.H., 1980. Principles and Procedures of Statistics. McGraw-Hill , New York.

Strauss, C.R., Wilson, B., Anderson, R. \& Williams, P.J., 1987. Development of precursors of $\mathrm{C}_{13}$ nor-isoprenoid flavorants in Riesling grapes. Am. J. Enol. Vitic. 38, 23-27.

Van Jaarsveld, F.P., Hattingh, S. \& Minnaar, P., 2009a. Rapid induction of ageing character in brandy products - Part II. Influence of type of oak. S. Afr. J. Enol. Vitic. 30, 16-23.

Van Jaarsveld, F.P., Hattingh, S. \& Minnaar, P., 2009b. Rapid induction of ageing character in brandy products - Part III. Influence of toasting. S. Afr. J. Enol. Vitic. 30, 24-37.

Van Jaarsveld, F.P., Minnaar, P., Blom, M. \& Hattingh, S., 2009. Rapid induction of ageing character in brandy products - Part I. Effects of extraction media and preparation conditions. S. Afr. J. Enol. Vitic. 30, 1-15.

Watts, V.A., Butzke, C.E. \& Boulton, R.B., 2003. Study of aged cognac using solid-phase microextraction and partial least-squares regression. J. Agr. Food Chem. 51, 7738-7742. 
Wondra, M. \& Berovic, M., 2001. Analyses of aroma components of chardonnay wine fermented by different yeast strains. Food Technol. Biotechnol. 39, 141148.

Zhao, Y.P., Li, J.M., Xu, Y., Fan, W.L. \& Jiang, W.G., 2009a. Characterization of aroma compounds of four brandies by aroma extract dilution analysis. Am. J. Enol. Vitic. 60, 269-277.
Zhao, Y.P., Xu, Y., Li, J.M., Fan, W.L. \& Jiang, W.G., 2009b. Profile of volatile compounds in 11 brandies by headspace solid-phase microextraction followed by gas chromatography-mass spectrometry. J. Food Sci. 74, C90C99. 